Check for updates

Cite this: RSC Adv., 2019, 9, 24760

\section{The removal mechanism and performance of tetrabromobisphenol A with a novel multi-group activated carbon from recycling long-root Eichhornia crassipes plants $\uparrow$}

\author{
Lili Liu, ${ }^{\text {ad }}$ Xin Chen, ${ }^{\text {a }}$ Zhiping Wang, ${ }^{\mathrm{c}}$ Xixi Wang ${ }^{\mathrm{a}}$ and Sen Lin (D) *abd
}

Long-root Eichhornia crassipes has shown great potential in eutrophication treatments while the heavy disposal of its plants limits its large-scale application. In this study, the adsorption of TBBPA by a novel multi-group activated carbon (MGAC), prepared from the reaped long-root Eichhornia crassipes plants has been investigated as a potential recycling and remediation technology. The MGAC showed great adsorption performance for aqueous TBBPA in that the adsorption could arrive at equilibrium in $4 \mathrm{~h}$ and the saturated adsorption capacities could reach up to $110.7,110.5$ and $75.50 \mathrm{mg} \mathrm{g}^{-1}$ at 20,30 and $40{ }^{\circ} \mathrm{C}$, respectively. Based on the analysis of adsorption processes, it was confirmed that $\pi-\pi$ interaction and hydrogen bonding were the major impetuses for the adsorption and the oxygen-containing functional groups on the MGAC surface could facilitate the adsorption by either electron sharing or electron transfer. In addition, the thermodynamic results showed that the adsorption was a spontaneous and exothermic reaction. Futhermore, the MGAC could be regenerated easily by $5 \% \mathrm{NaOH}$ solution and retained over $50 \%$ of its initial capacities for TBBPA after 5 reprocessing cycles. These results indicate the promising application of MGAC in the wastewater treatment for TBBPA removal and a resource recycling method for the long-root Eichhornia crassipes plants.
Received 6th May 2019 Accepted 30th July 2019 DOI: $10.1039 / c 9 r a 03374 b$ rsc.li/rsc-advances extensively investigated methods due to its cheapness, effectiveness and convenience for subsequent operation.

At the same time, the high cost of raw materials (coconut shell, wood, coal, etc.) for commercial activated carbon leads to the high price of large-scale wastewater treatment using activated carbon. Therefore, the preparation of activated carbon from plant materials in natural environment has the advantages of low cost, environmental friendliness and rich structures. Among them, long-root Eichhornia crassipes with the characteristics of rapid growth and reproduction, wide distribution and easy access, has been planted to solve the eutrophication in Dianchi Lake in China dependent on its ultralong root, while how to dispose these ripe plants is still a great challenge. Therefore, it's essential and of great environmental significance to explore available approaches for the recycle of waste longroot Eichhornia crassipes plants for the practical applicability of this eutrophication treatment technology. According to the characteristics of the long-root Eichhornia crassipes, it would be comparatively profitable to transform and recycle them into the high-performance activated carbon for environmental contaminants. At present, most of the studies were focused on the adsorption of heavy metals by long-root Eichhornia crassipes activated carbon, while there are few reports on the adsorption of organic pollutants, which limited its further promotion..$^{21-23}$ Therefore, it is necessary to increase the research on the

\footnotetext{
${ }^{a}$ State Environmental Protection Key Laboratory of Environmental Risk Assessment and Control on Chemical Process, East China University of Science and Technology, Shanghai, 200237, China. E-mail: linsen@ecust.edu.cn

${ }^{b}$ National Engineering Research Center for Integrated Utilization of Salt Lake Resources, East China University of Science and Technology, Shanghai, China

${ }^{c}$ School of Environment Science and Technology, Shanghai Jiao Tong University, Shanghai, China

${ }^{d}$ Shanghai Institute of Pollution Control and Ecological Security, Shanghai, China

$\dagger$ Electronic supplementary information (ESI) available. See DOI: 10.1039/c9ra03374b
} 
adsorption mechanism of organic matters. In addition, it is extremely necessary to understand the underlying adsorption processes and mechanisms for the adsorption to further improving the practical performance of adsorbents.

In this study, a novel multi-group activated carbon (MGAC) was prepared from the long-root Eichhornia crassipes to investigate the adsorption performance and specific mechanism for aqueous TBBPA in the first time. After the preparation, the physicochemical characteristics of the MGAC were determined. Then the effects of different exogenous factors on adsorption were explored to deduce the correlative impetuses, including initial $\mathrm{pH}$, ionic strength, etc. In addition, the adsorption dynamics and thermodynamics were described by multifarious models to analyze adsorption performance and mechanism. Finally, the regeneration and recycling of the MGAC were also investigated. Based on these results, the in-depth understanding of the adsorption mechanism and demonstration of the adsorption, regeneration and reuse of the MGAC was obtained and improved. It further indicated the feasibility of the practical application and recycling utilization of long-root Eichhornia crassipes plants.

\section{Materials and methods}

\subsection{Chemicals}

TBBPA used for adsorption was purchased from J\&K Scientific Co., Ltd (Shanghai, China) with the purity over $98.0 \%$. The other chemicals used in this work were all of analytically reagent grade and no further purification was performed prior to use. The TBBPA stock solution was prepared by dissolving $100 \mathrm{mg}$ TBBPA into $100 \mathrm{ml} 0.1 \%(\mathrm{~m} / \mathrm{m}) \mathrm{NaOH}$ solution. This solution was further diluted with deionized water to the concentration required for the following experiments. The long-root Eichhornia crassipes plants used to prepare the MGAC were reaped from the Dianchi Lake, Yunnan, China. In all experiments, the initial $\mathrm{pH}$ values of the solutions were adjusted by using $1 \% \mathrm{HCl}$ or $1 \%$ $\mathrm{NaOH}$ solution.

\subsection{Preparation of the MGAC}

$10.0 \mathrm{~g}$ powder of long-root Eichhornia crassipes, which had been dried and grinded, mixed with $30 \mathrm{ml} 25 \% \mathrm{KOH}$ solution under stirring for primary carbonization. Then $100 \mathrm{ml}$ deionized water was added to impregnate the powder for $12 \mathrm{~h}$. Afterwards, the powder was calcined at $600{ }^{\circ} \mathrm{C}$ for $1 \mathrm{~h}$ with nitrogen protection after dried at $100{ }^{\circ} \mathrm{C}$. Finally, the MGAC could be collected by wash with $5 \% \mathrm{HCl}$ to activate the calcined product.

\subsection{Adsorption experiments}

For a typical batch experiment, $15 \mathrm{mg}$ MGAC was added to $100 \mathrm{ml}$ TBBPA work solution with selected concentration at a given $\mathrm{pH}$ in a $250 \mathrm{ml}$ flask. The flask was then placed in a temperature-controlled incubator shaker (YRH-300, Yaoshi Instrument, China) at $175 \mathrm{rpm}$ at a given temperature. The supernatant was sampled at specified time intervals and filtered through a $0.22 \mu \mathrm{m}$ filter for analysis. The adsorption capacity was calculated according to eqn (1).

$$
q_{\mathrm{e}}=\left(C_{0}-C_{\mathrm{e}}\right) \times V / m
$$

where $C_{0}\left(\mathrm{mg} \mathrm{L}^{-1}\right)$ and $C_{\mathrm{e}}\left(\mathrm{mg} \mathrm{L}^{-1}\right)$ were the initial and equilibrium concentrations of the target contaminant, respectively. And $q_{\mathrm{e}}\left(\mathrm{mg} \mathrm{g}^{-1}\right)$ was the adsorption capacity of the MGAC at equilibrium. $V(L)$ was the contaminant solution volume and $m$ (g) was the mass of the MGAC.

All experiments were performed in triplicate and the variations between parallel experiments were less than 5\%. All glassware used were cleansed via sonication at $40 \mathrm{kHz}$ for $30 \mathrm{~min}$ and then dried out before use.

\subsection{Adsorption kinetics}

The adsorption kinetic models were used to describe the adsorption process and the effect of contact time. $15 \mathrm{mg}$ MGAC mixed with $100 \mathrm{ml}$ TBBPA solutions of concentrations 5, 10 and $20 \mathrm{mg} \mathrm{L}^{-1}$ in $250 \mathrm{ml}$ flask at pH 9.0, $175 \mathrm{rpm}$ and $30{ }^{\circ} \mathrm{C}$. The supernatant was sampled at specified time intervals and filtered through a $0.22 \mu \mathrm{m}$ filter for analysis. All experiments were performed in triplicate and the variations between parallel experiments were less than $5 \%$.

The pseudo-first-order and pseudo-second-order kinetic models can be expressed as (2) and (3), respectively:

$$
\begin{gathered}
q_{t}=q_{\mathrm{e}}\left(1-\mathrm{e}^{-k_{1} t}\right) \\
q_{t}=k_{2} q_{\mathrm{e}}^{2} t /\left(1+k_{2} q_{\mathrm{e}} t\right)
\end{gathered}
$$

where $q_{t}\left(\mathrm{mg} \mathrm{g}^{-1}\right)$ is the adsorbed amount at time $t(\mathrm{~h}), q_{\mathrm{e}}(\mathrm{mg}$ $\left.\mathrm{g}^{-1}\right)$ is the adsorbed amount at equilibrium, $k_{1}\left(\mathrm{~h}^{-1}\right), k_{2}(\mathrm{~g}(\mathrm{mg}$ $\mathrm{h})^{-1}$ ) are the pseudo-first-order and pseudo-second-order rate constant, respectively.

The intra-particle diffusion equation is:

$$
q_{t}=k_{\mathrm{ip}} t^{1 / 2}+C
$$

where $k_{\mathrm{ip}}\left[\mathrm{mg}\left(\mathrm{g} \mathrm{h}^{1 / 2}\right)^{-1}\right]$ is the rate constant in the intra-particle diffusion equation. The boundary layer thickness is determined by $C$. If the plot of $q_{t}\left(\mathrm{mg} \mathrm{g}^{-1}\right) v s . t^{1 / 2}\left(\mathrm{~h}^{1 / 2}\right)$ is a straight line and passes through the origin, the sole factor controlling the adsorption ratio is intra-particle diffusion. ${ }^{24}$

\subsection{Adsorption isotherms}

The adsorption isotherm models were used to determine the adsorption mechanism and describe how TBBPA molecules interact with the MGAC. $100 \mathrm{ml}$ TBBPA solutions of five concentrations, i.e., 2, 5, 10, 20, 30 $\mathrm{mg} \mathrm{L}^{-1}$, were prepared and used in adsorption isotherm experiments. The supernatant was sampled at specified time intervals and filtered through a 0.22 $\mu \mathrm{m}$ filter for analysis. All experiments were performed in triplicate and the variations between parallel experiments were less than $5 \%$.

Langmuir isotherm assumes that the adsorbate is uniformly adsorbed on the surface of the adsorbent. The adsorption process is monolayer and the adsorbate does not move on the surface. 


$$
C_{\mathrm{e}} / Q_{\mathrm{e}}=1 /\left(Q_{\mathrm{m}} K_{\mathrm{L}}\right)+C_{\mathrm{e}} / Q_{\mathrm{m}}
$$

where $Q_{\mathrm{e}}\left(\mathrm{mg} \mathrm{g}^{-1}\right)$ is the equilibrium adsorbance, $C_{\mathrm{e}}\left(\mathrm{mg} \mathrm{L}^{-1}\right)$ is the equilibrium concentration of TBBPA in the solution, $Q_{\mathrm{m}}(\mathrm{mg}$ $\left.\mathrm{g}^{-1}\right)$ is the saturation adsorption capacity, $K_{\mathrm{L}}\left(\mathrm{L} \mathrm{mg}^{-1}\right)$ is the Langmuir model constant.

The Freundlich models indicates that multilayer adsorption is carried out on a heterogeneous surface, which was expressed by eqn (6).

$$
\ln Q_{\mathrm{e}}=\ln K_{\mathrm{F}}+\frac{1}{n} \ln C_{\mathrm{e}}
$$

where $Q_{\mathrm{e}}\left(\mathrm{mg} \mathrm{g}^{-1}\right)$ is the equilibrium adsorbance, $C_{\mathrm{e}}\left(\mathrm{mg} \mathrm{L}^{-1}\right)$ is the equilibrium concentration of TBBPA in the solution, $Q_{\mathrm{m}}(\mathrm{mg}$ $\mathrm{g}^{-1}$ ) is the saturation adsorption capacity, $K_{\mathrm{F}}$ and $n$ are the Freundlich model constants.

The Temkin model is mainly used to describe the chemical adsorption process. It is considered that the adsorption heat varies linearly with temperature. The equation is as follows:

$$
Q_{\mathrm{e}}=B_{1} \ln K_{\mathrm{T}}+B_{1} \ln C_{\mathrm{e}}
$$

where $Q_{\mathrm{e}}\left(\mathrm{mg} \mathrm{g}^{-1}\right)$ is the equilibrium adsorbance, $C_{\mathrm{e}}\left(\mathrm{mg} \mathrm{L}^{-1}\right)$ is the equilibrium concentration of TBBPA in the solution, $B_{1}$ is the reaction heat of adsorption, $K_{\mathrm{T}}\left(\mathrm{L} \mathrm{mg}^{-1}\right)$ is the Temkin model constants.

The Dubinin-Radushkevich (D-R) model equation is as follows:

$$
\begin{aligned}
& \ln Q_{\mathrm{e}}=\ln Q_{\mathrm{m}}-k \varepsilon^{2} \\
& \varepsilon=R T \ln \left(1+1 / C_{\mathrm{e}}\right)
\end{aligned}
$$

where $Q_{\mathrm{e}}\left(\mathrm{mg} \mathrm{g}^{-1}\right)$ is the equilibrium adsorbance, $C_{\mathrm{e}}\left(\mathrm{mg} \mathrm{L}^{-1}\right)$ is the equilibrium concentration of TBBPA in the solution, $k\left(\mathrm{~mol}^{2}\right.$ $\mathrm{J}^{-2}$ ) is a constant that corresponds to the adsorption energy, $\varepsilon$ is the Polanyi potential, $R$ is the ideal gas constant $[8.314 \mathrm{~J}(\mathrm{~mol}$ $\left.\mathrm{K})^{-1}\right], Q_{\mathrm{m}}\left(\mathrm{mg} \mathrm{g}^{-1}\right)$ is the maximum adsorption capacity.

In addition, the adsorption thermodynamics parameters including standard enthalpy $\left(\Delta H^{0}, \mathrm{~kJ} \mathrm{~mol}^{-1}\right)$, standard entropy $\left(\Delta S^{0}, \mathrm{~J}(\mathrm{~mol} \mathrm{~K})^{-1}\right)$ and Gibbs free energy $\left(\Delta G^{0}, \mathrm{~kJ} \mathrm{~mol}^{-1}\right)$ were calculated according to eqn (10) and (11).

$$
\begin{gathered}
\ln \mathrm{K}=\frac{\Delta S^{0}}{R}-\frac{\Delta H^{0}}{R T} \\
\Delta G^{0}=\Delta H^{0}-T \Delta S^{0}
\end{gathered}
$$

where $K\left(\mathrm{~L} \mathrm{~g}^{-1}\right)$ is the adsorption equilibrium constant, $R(8.314$ $\mathrm{J}(\mathrm{mol} \mathrm{K})^{-1}$ ) is the universal gas constant, $T$ is the temperature (K).

\subsection{Regeneration}

$1.0 \mathrm{~g}$ of the used MGAC mixed with $30 \mathrm{ml} 5 \% \mathrm{NaOH}$ solution in a $150 \mathrm{ml}$ flask and then the flask was placed in incubator shaker at $30^{\circ} \mathrm{C}$ and $175 \mathrm{rpm}$ for $2 \mathrm{~h}$. At last the spent MGAC was washed with deionized water until the solution $\mathrm{pH}$ reached 6.0-7.0. After the regeneration, the remaining TBBPA in the solution was measured, and the regenerated MGAC was applied for the subsequent adsorption cycles.

\subsection{Characterization methods}

The TEM and SEM images of the MGAC were examined on an transmission electron microscope (JEM-2100, JEOL, Japan) with a high voltage of $200 \mathrm{kV}$ and an scanning electron microscopy (JSM-6360LV, JEOL, Japan) with $5.0 \mathrm{kV}$, respectively. The specific surface area was calculated by the Brunauer-Emmett-Teller (BET) method and the pore volume and pore size distribution were estimated based on Barrett-Joyner-Halenda (BJH) model. The X-ray photoelectron spectroscopy (XPS) spectrum of this adsorbent was obtained by an XPS instrument (ESCALAB 250Xi, Thermo Scientific, USA) with the Al-KR as the excitation source. The zeta potentials of the MGAC were determined by a zeta potential instrument (Nano ZS, Malvern, UK) following the method of Wang et $a .^{25}$ Fourier-transform infrared (FTIR) spectra of the MGAC before and after adsorption were recorded from samples in the wavenumber range of $4000-400 \mathrm{~cm}^{-1}$ in $\mathrm{KBr}$ pellets on a FTIR spectrometer (NICOLET 6700, Thermofisher, USA). The concentrations of TBBPA in solutions were determined by a high performance liquid chromatography (HPLC) (LC-20AT, Shimadzu, Japan) equipped with an UV detector and a C18 reverse-phase column (Inertsil ODS) $(250 \mathrm{~mm} \times 4.6 \mathrm{~mm}$ i.d., particle size $5 \mu \mathrm{m})$ using methanol/ water $(80: 20(\mathrm{v} / \mathrm{v}))$ mobile phase. The $\mathrm{pH}$ values of solutions were measured with a $\mathrm{pH}$ meter (HQ30d, HACH, USA) after calibration.

\section{Results and discussion}

\subsection{Characterization of the MGAC}

In this study, multiple measurements were applied to characterize the physicochemical properties of the prepared MGAC. Firstly, the morphology and microstructure of the MGAC were demonstrated by the TEM and SEM images (Fig. 1). The TEM observation of the MGAC indicated that the carbon had significantly hierarchical porous structures with honeycomblike shape (Fig. 1a). And the SEM (Fig. 1b) observation presented a rough and irregular surface with pores on the MGAC. According to the BET result, it might be related to the porosity of the adsorbent, thus resulted in a high specific surface area ${ }^{26,27}$ reaching than $874.3 \mathrm{~m}^{2} \mathrm{~g}^{-1}$. The pore size distribution curve (Fig. 1c) exhibited in the category of mesopore, with the pore volume and average size being at $0.38 \mathrm{~cm}^{3} \mathrm{~g}^{-1}$ and $6.96 \mathrm{~nm}$, respectively. In addition, the isoelectric point of the MGAC was identified from variation trend of the zeta potential as a function of $\mathrm{pH}$ values. As shown in Fig. 1d, the zeta potential decreased with the increasing $\mathrm{pH}$, from which the isoelectric point was determined as 3.3, indicating the MGAC surface was electronegative in non-acid condition.

To further understand the functional groups on the MGAC surface, the wide XPS spectrum of the MGAC was surveyed (Fig. S1 $\dagger$ ), implying the oxygen, carbon and nitrogen were the dominant elements. To further explore the surface carbon and oxygen functional components, the high-resolution spectrum of 

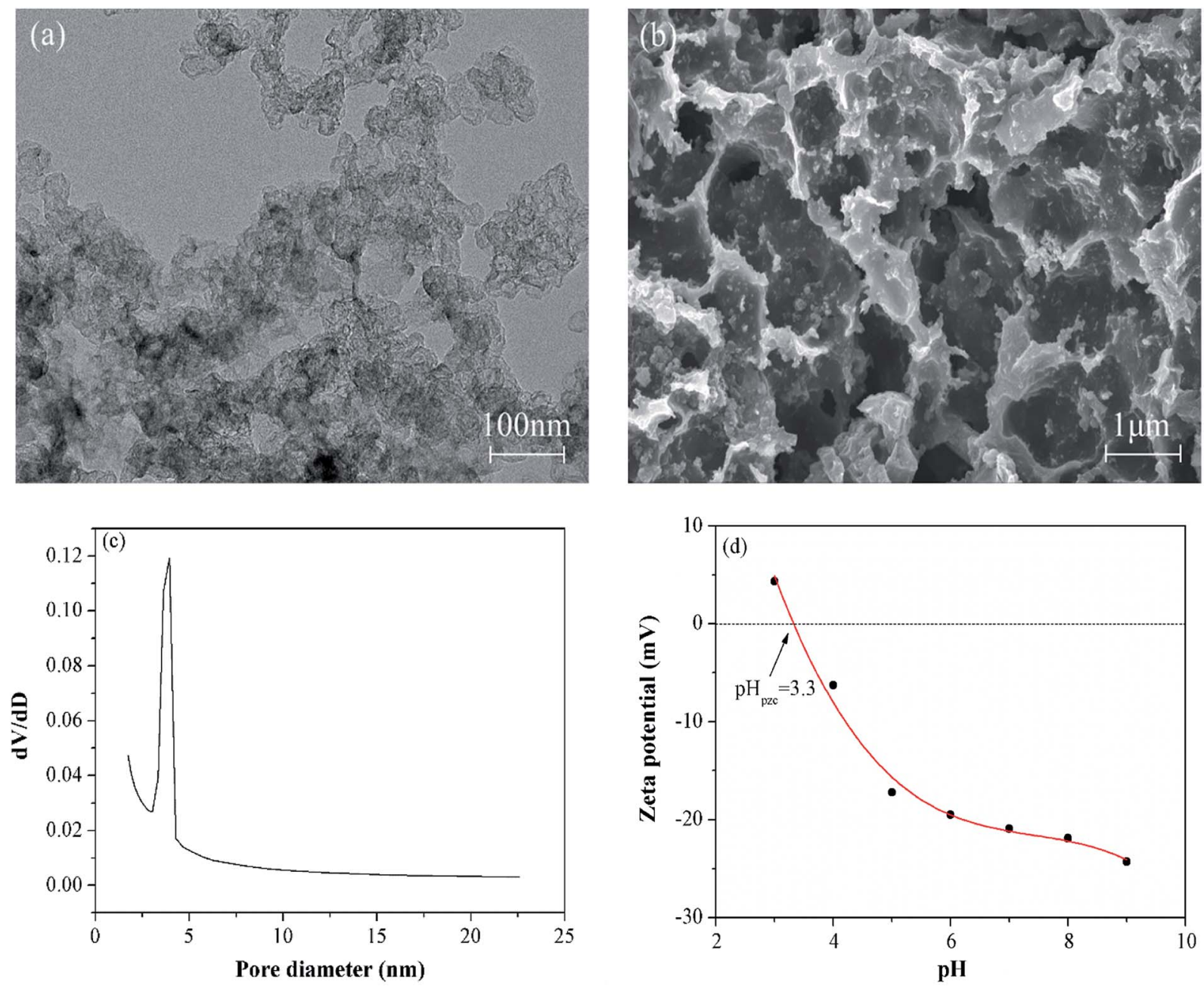

Fig. 1 The SEM (a) and TEM (b) images of the MGAC; the pore size distribution (c) of the MGAC; the zeta potential of the MGAC as a function of $\mathrm{pH}(\mathrm{d})$.

C1s and O1s were deconvoluted by XPSPEAK41 software and the results were plotted in Fig. 2. And the C1s peak could be decomposed into four individual peaks at 284.6, 285.4, 286.6 and $289.2 \mathrm{eV}$ (Fig. 2a), which were responsible for $\mathrm{C}-\mathrm{C}$ in aromatic rings, $\mathrm{C}-\mathrm{C}=\mathrm{O}, \mathrm{C}-\mathrm{O}$ and $\mathrm{O}-\mathrm{C}=\mathrm{O}$, respectively. ${ }^{28,29} \mathrm{In}$ the case of oxygen in Fig. $2 \mathrm{~b}$, there were also two distinct oxygen
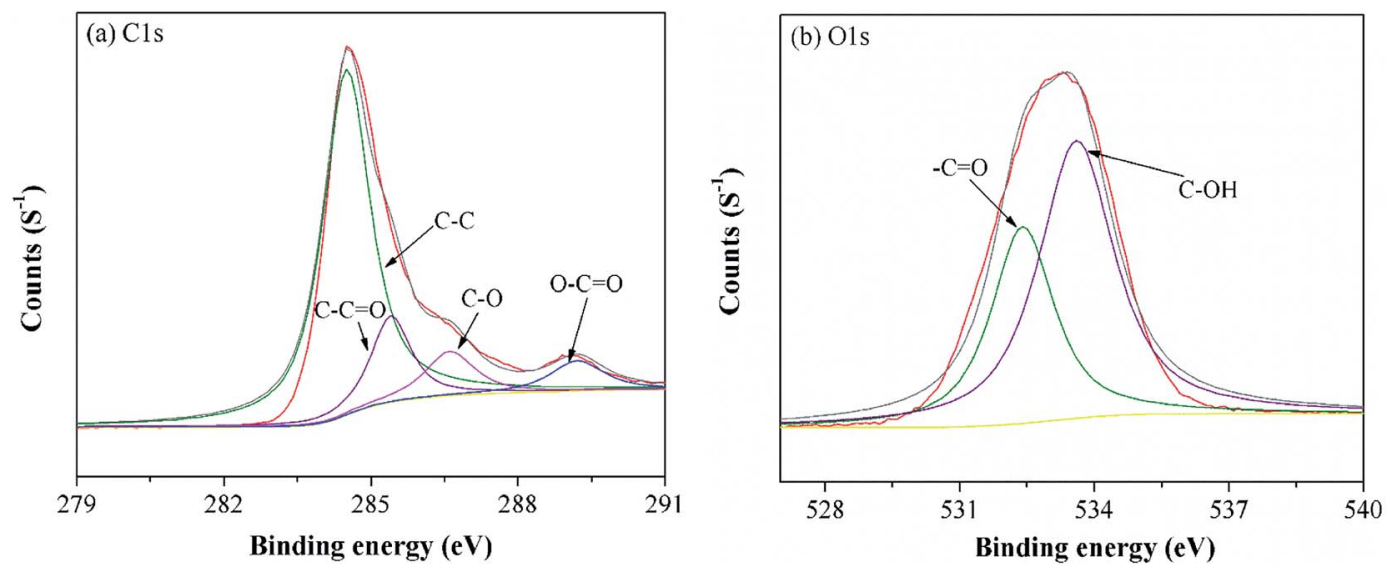

Fig. 2 High resolution XPS spectra of C1s (a) and O1s (b) on the MGAC surface. 


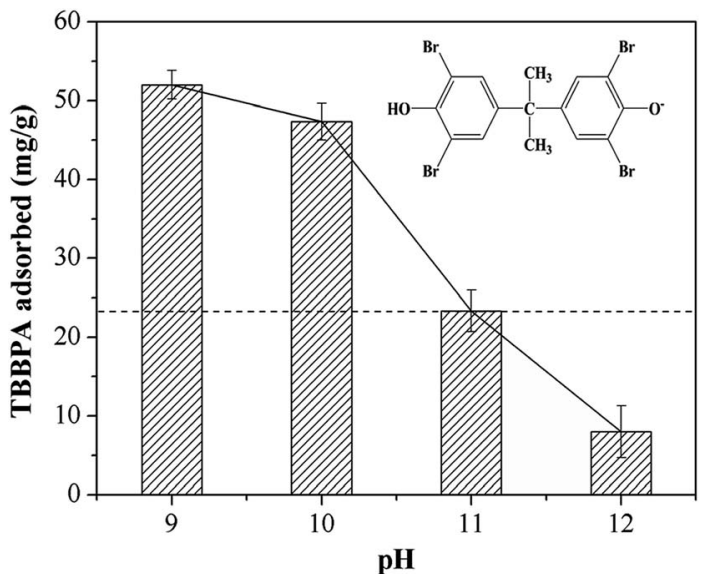

Fig. 3 Effect of initial $\mathrm{pH}$ on TBBPA adsorption using the MGAC (TBBPA $=10 \mathrm{mg} \mathrm{L}^{-1}, \mathrm{MGAC}=150 \mathrm{mg} \mathrm{L}^{-1}, T=30^{\circ} \mathrm{C}, t=10 \mathrm{~h}$ ).

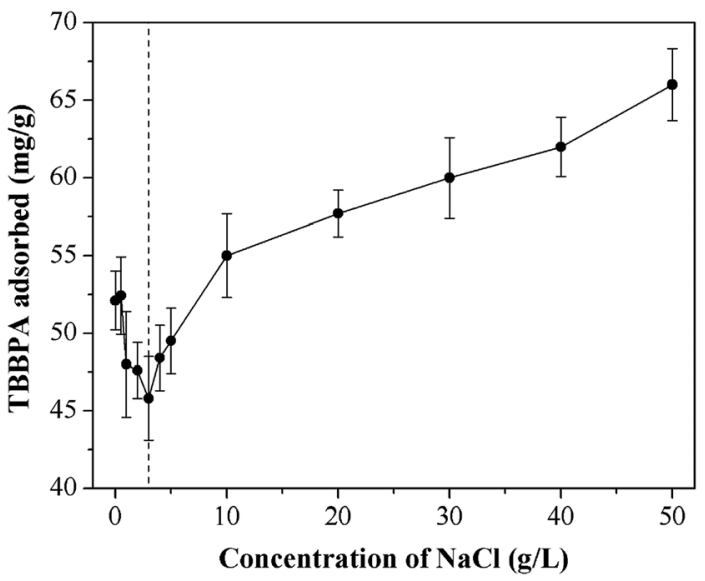

Fig. 4 Effect of ionic strength on TBBPA adsorption using the MGAC (TBBPA $=10 \mathrm{mg} \mathrm{L}^{-1}, \mathrm{MGAC}=150 \mathrm{mg} \mathrm{L}^{-1}, T=30^{\circ} \mathrm{C}, \mathrm{pH}=9.0, t=10$ h).

bands at 532.4, and $533.6 \mathrm{eV}$, which were ascribed to $\mathrm{C}-\mathrm{O}$ and $\mathrm{C}-\mathrm{OH}$, respectively, consistent with previous work. ${ }^{\mathbf{1 4}}$ The proportions of functional groups both in C1s and O1s peak indicated that there were abundant oxygen-containing functional groups on the MGAC surface, affording great potential for the adsorption (Table $\mathrm{S} 1 \dagger$ ).

\subsection{Effects of environmental factors on the adsorption of TBBPA}

3.2.1 Initial pH. $\mathrm{pH}$ is one of the vital factors affecting the adsorption performance, which determines the surface charge on the adsorbent surface as well as the existing speciation of adsorbate in solution. The experiments were conducted with initial $\mathrm{pH}$ value varying in the range of 9.0-12.0, in view of the insolubility of TBBPA in non-alkaline solution. ${ }^{30}$ As shown in Fig. 3, TBBPA adsorbed on the MGAC decreased from $52.1 \mathrm{mg}$ $\mathrm{g}^{-1}$ to $8.0 \mathrm{mg} \mathrm{g}^{-1}$ with the increase of initial $\mathrm{pH}$ in solution from 9.0 to 12.0 , indicating the alkaline condition unfavorable for the adsorption. According to the distribution of molecular and anionic forms as a function of $\mathrm{pH}$, illustrated in Fig. S2, $\uparrow$ TBBPA was mostly ionized to mono or divalent anions in the alkaline condition. ${ }^{18}$ As the $\mathrm{pH}$ value exceeded 9.0, these functional groups on the MGAC surface were deprotonated, such as $-\mathrm{COO}^{-},-\mathrm{O}^{-}$, etc., resulting in the decrease of the adsorption capacity due to the enhanced electrostatic repulsion between the MGAC surface and TBBPA. In addition, attributed to the reduction of hydrogen bonding in strongly basic solution, which was prevented by the enhanced charge repulsion, TBBPA adsorption decreased severely when $\mathrm{pH}$ increased from 10.0 to 12.0.

3.2.2 Ionic strength. In this study, the effects of ionic strength on TBBPA adsorption using the MGAC was performed with the concentrations of $\mathrm{NaCl}$ at the range of $0-50 \mathrm{~g} \mathrm{~L}^{-1}$. The adsorption capacity decreased slightly with $\mathrm{NaCl}$ concentration in the range of $0-3 \mathrm{~g} \mathrm{~L}^{-1}$ (Fig. 4), due to that the electrostatic repulsion would be reinforced under a small amount of $\mathrm{NaCl}$ in solution. On the contrary, the adsorption capacity of TBBPA increased from $48.4 \mathrm{mg} \mathrm{g}^{-1}$ to $66.0 \mathrm{mg} \mathrm{g}^{-1}$ with the increase of $\mathrm{NaCl}$ concentration in 3-50 $\mathrm{mg} \mathrm{L}^{-1}$. According to the results of Amrit et al.,$^{31}$ the increased adsorption capacity might come from the $\pi-\pi$ interaction between TBBPA and the MGAC, which could be effectively enhanced by stronger ionic strength. In addition, $\mathrm{Na}^{+}$bridged with negatively charged surface groups might promote the adsorption resulting from offsetting the negative effects of competing adsorption sites. ${ }^{32}$

3.2.3 Humic acid. Humic acid (HA) is ubiquitous in natural waters derived by the microbial degradation of dead plants, which is always concomitant with TBPPA. ${ }^{33}$ Hence, it's full of importance to investigate the effect of the coexistence of HA on TBBPA adsorption. According to results of TBBPA adsorption at different concentrations of HA (Fig. 5), the adsorption capacity decreased significantly with HA concentration less than $10 \mathrm{mg} \mathrm{L}^{-1}$ while the adsorbed TBBPA was enhanced as HA concentration continued to increase. The basic structure of HA is aromatic and alicyclic ring, connected with carboxyl, carbonyl, quinonyl, hydroxyl and methoxy groups. ${ }^{34}$ All these structure can form the $\pi-\pi$ interaction, hydrogen bonding with MGAC, which could attributed to the competitive adsorption of HA and TBBPA on adsorbents thus reduce the removal efficiency of TBBPA. ${ }^{35,36}$ The slight increase in adsorption at high concentration of HA was presumably due to the adsorption of free TBBPA to HA which was sorbed to the MGAC surface. ${ }^{37}$

\subsection{Adsorption kinetics}

The adsorption kinetics of TBBPA using the MGAC was conducted under the initial concentrations of 5, 10 and $20 \mathrm{mg} \mathrm{L}^{-1}$. As shown in Fig. 6, it could be seen that the TBBPA adsorped on the MGAC surface all increased quickly in the first $2 \mathrm{~h}$ owing to the rich availability of active sites, and then the adsorption rate slowed down until the adsorption equilibrium reached in $4 \mathrm{~h}$. In order to further analyze the adsorption processes, the pseudofirst-order and pseudo-second-order kinetic models were both used to fit the adsorption kinetic data shown in Fig. 6a. It could been seen that the adsorption was all better described by the pseudo-second-order model for different initial concentrations, 


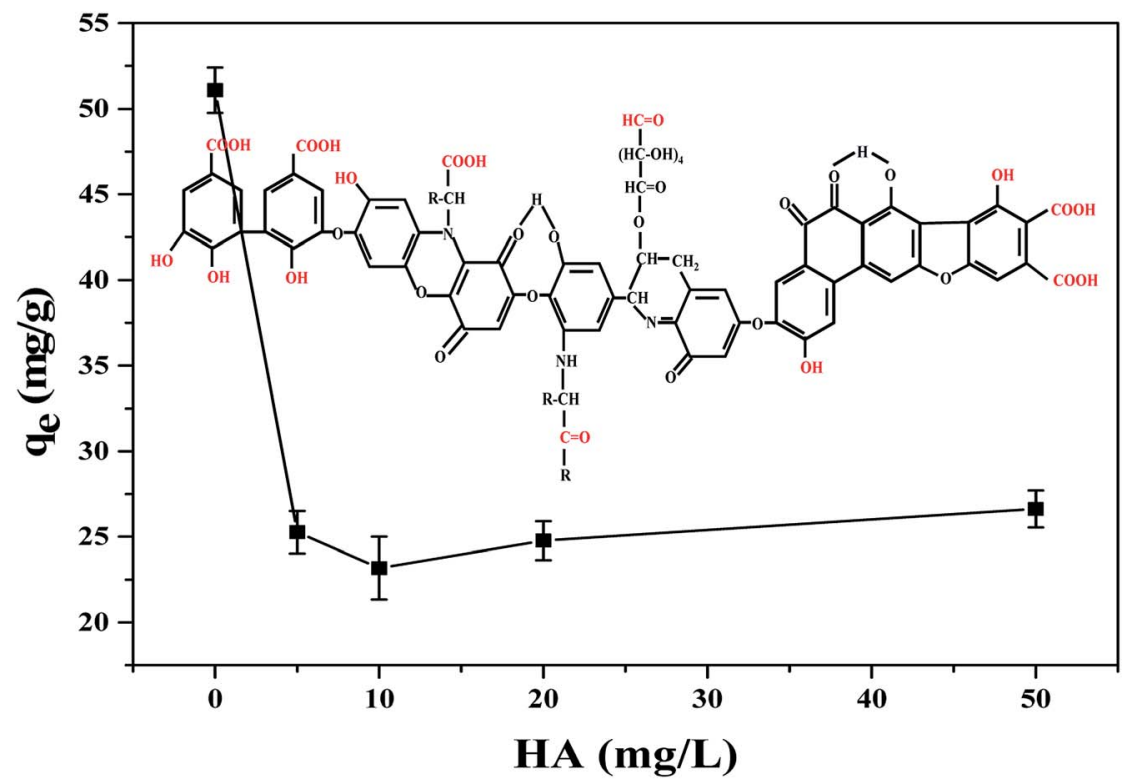

Fig. 5 Effect of $\mathrm{HA}$ on TBBPA adsorption using the MGAC (TBBPA $=10 \mathrm{mg} \mathrm{L}^{-1}, \mathrm{MGAC}=150 \mathrm{mg} \mathrm{L}^{-1}, \mathrm{HA}=0,5,10,20,50 \mathrm{mg} \mathrm{L}-1, T=30^{\circ} \mathrm{C}, \mathrm{pH}$ $=9.0, t=10 \mathrm{~h}$ ).

which was verified by the fitting coefficients listed in Table 1 higher than 0.96. According to the hypothesis of the pseudosecond-order model, it indicated that the adsorption rate was controlled by chemical interaction, and there might be electron exchange or covalent bond between adsorbents and adsorbates. Besides the adsorption capacity was proportional to the functional groups involving $\pi-\pi$ bonding and hydrogen bonding interactions on the absorbent, consistent with the studies of Zhang et al. ${ }^{18}$

The plots of $q_{t} v s . t^{1 / 2}$ at different initial concentrations are shown in Fig. 6b. It could been seen that the plots are not linear and do not pass through the origin, which demonstrated that the adsorption was a complicated process including multiple steps. ${ }^{38}$ Fig. $6 \mathrm{~b}$ clearly shown that the adsorption could be divided into three steps. The first step was the instantaneous adsorption or external surface adsorption in the range of $0-0.5 \mathrm{~h}$ and approximately $50 \%$ of the adsorption capacities finished in this step mainly relying on the high concentration of TBBPA as the adsorption impetus. ${ }^{39}$ The second step was controlled by the intra-particle diffusion and the curve represented gradual adsorption, where TBBPA was removed by the internal sites. ${ }^{24}$ In the final equilibrium stage, the adsorption growth rate was almost constant and TBBPA concentration kept at a low level, indicating the adsorption equilibrium arriving. ${ }^{40}$

\subsection{Adsorption thermodynamics}

To further understand the adsorption mechanisms of TBBPA by the MGAC, adsorption isotherms were determined at temperature being at 20,30 and $40{ }^{\circ} \mathrm{C}$ with various models of Langmuir,
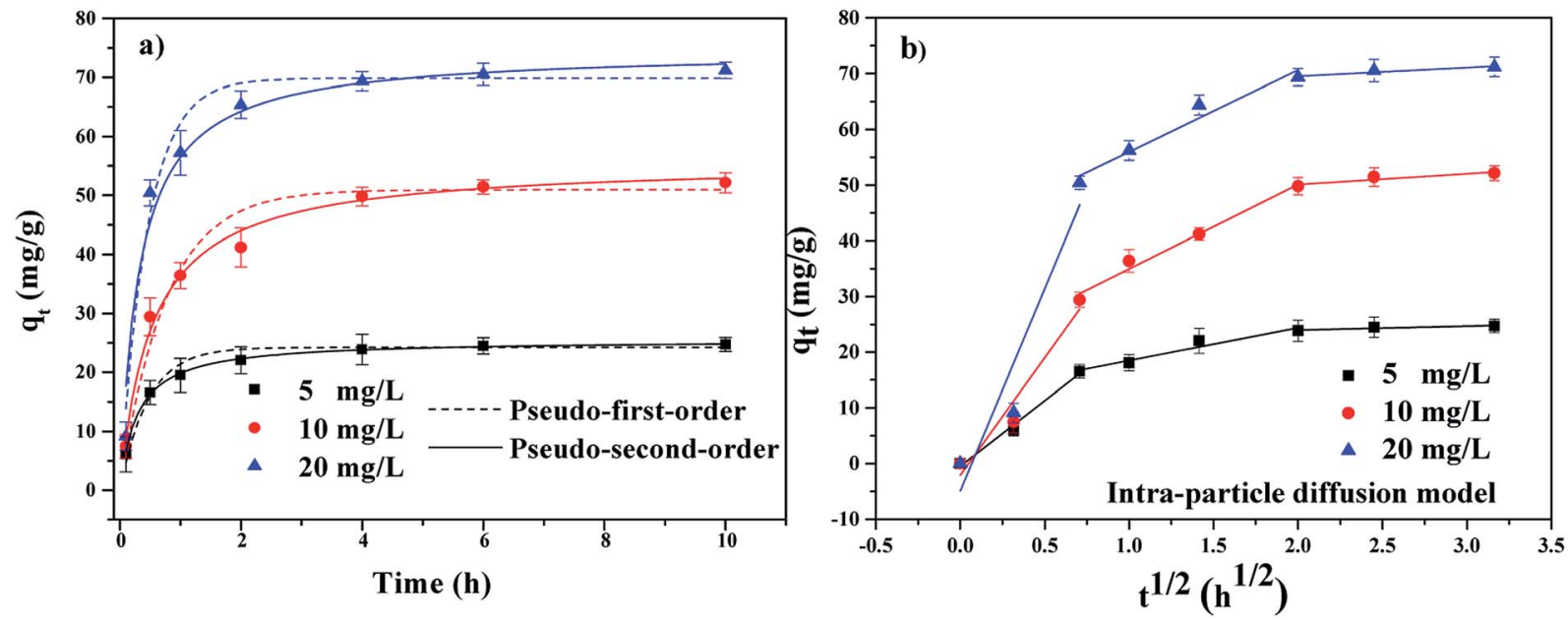

Fig. 6 The adsorption kinetics of TBBPA on the MGAC (TBBPA $=5,10$ and $20 \mathrm{mg} \mathrm{L}^{-1}, \mathrm{MGAC}=150 \mathrm{mg} \mathrm{L}^{-1}, \mathrm{~T}=30{ }^{\circ} \mathrm{C}, \mathrm{pH}=9.0, t=10 \mathrm{~h}$ ). 
Table 1 Kinetic fitting parameters for TBBPA adsorption on the MGAC

\begin{tabular}{lllllll}
\hline & \multicolumn{2}{l}{ Pseudo-first-order } & & & \multicolumn{2}{l}{ Pseudo-second-order } \\
\cline { 2 - 3 }$C_{0}\left(\mathrm{mg} \mathrm{L}^{-1}\right)$ & $k_{1}\left(\mathrm{~h}^{-1}\right)$ & $q_{\mathrm{e}}\left(\mathrm{mg} \mathrm{g}^{-1}\right)$ & $R^{2}$ & & $k_{2} \mathrm{~g}(\mathrm{mg} \mathrm{h})^{-1}$ & $q_{\mathrm{e}}\left(\mathrm{mg} \mathrm{g}^{-1}\right)$ \\
\hline 5 & 2.164 & 24.20 & 0.9567 & 0.137 & 25.48 \\
10 & 1.329 & 50.93 & 0.9800 & 0.033 & 0.9974 \\
20 & 2.223 & 69.84 & 0.9559 & 0.041 & 0.9940 \\
\hline
\end{tabular}

Intra-particle diffusion model

\begin{tabular}{|c|c|c|c|c|c|c|c|c|c|}
\hline$C_{0}\left(\mathrm{mg} \mathrm{L}^{-1}\right)$ & $K_{\text {ip } 1}\left(\mathrm{mg} \mathrm{g}^{-1} \mathrm{~h}^{-0.5}\right)$ & $C_{1}\left(\mathrm{mg} \mathrm{g}^{-1}\right)$ & $R^{2}$ & $K_{\text {ip } 2}\left(\mathrm{mg} \mathrm{g}^{-1} \mathrm{~h}^{-0.5}\right)$ & $C_{2}\left(\mathrm{mg} \mathrm{g}^{-1}\right)$ & $R^{2}$ & $K_{\text {ip } 3}\left(\mathrm{mg} \mathrm{g}^{-1} \mathrm{~h}^{-0.5}\right)$ & $C_{3}\left(\mathrm{mg} \mathrm{g}^{-1}\right)$ & $R^{2}$ \\
\hline 10 & 42.1574 & -2.0828 & 0.9085 & 15.1477 & 19.8061 & 0.9766 & 1.9223 & 46.2472 & 0.7555 \\
\hline 20 & 72.6213 & -4.9208 & 0.8346 & 14.6827 & 41.2785 & 0.9382 & 1.5370 & 66.4384 & 0.8304 \\
\hline
\end{tabular}

Freundlich, Temkin and D-R equations, respectively (Fig. 7). The corresponding models and fitting parameters were given in Table 2. Among the different models, the Langmuir model, with the higher correlation coefficient values $\left(R^{2}>0.981\right)$, could describe the adsorption better than the other isotherm models, indicating the existence of monolayer adsorption in the TBBPA
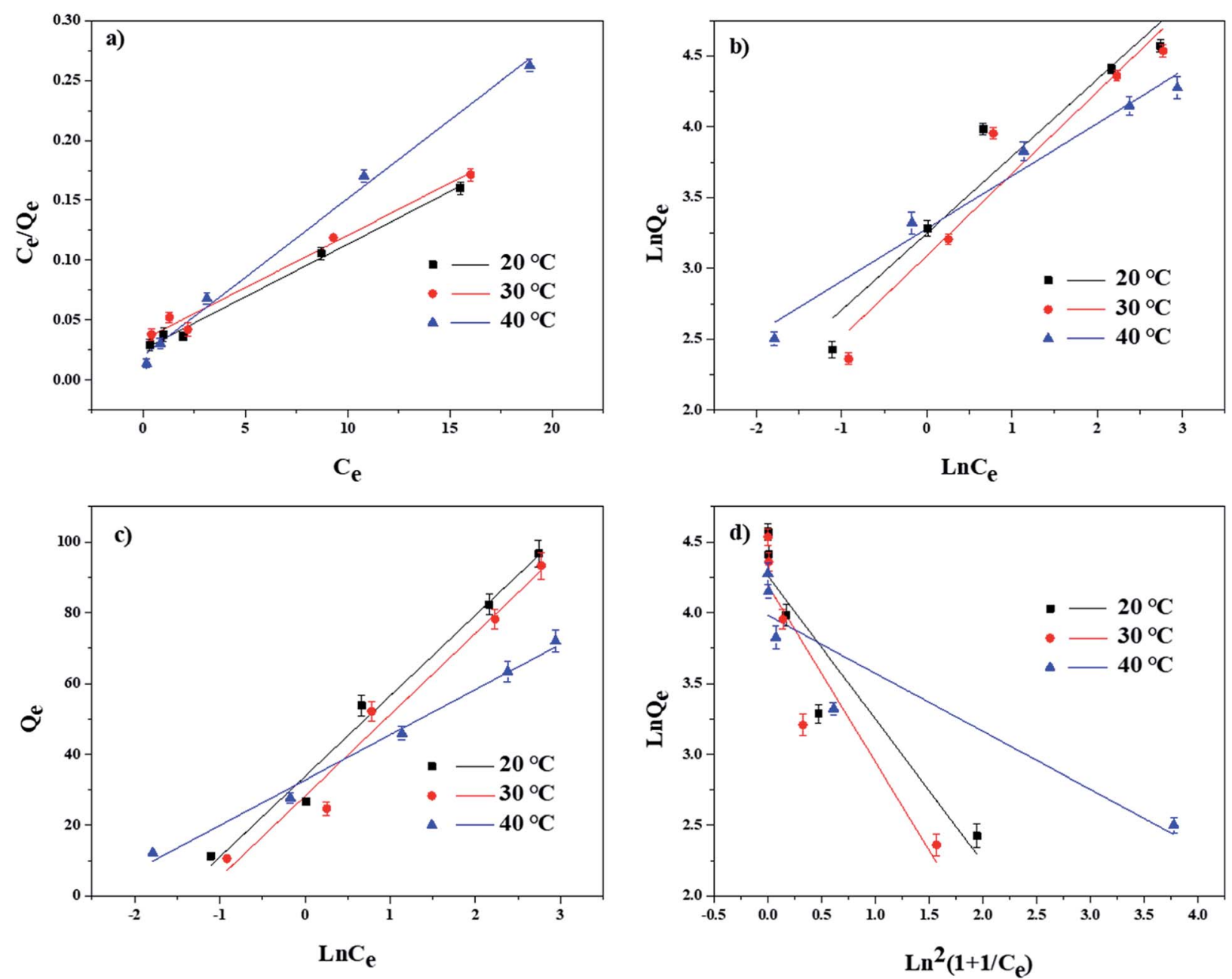

Fig. 7 Adsorption isotherms of TBBPA on MGAC in different temperature as well as modeling using the (a) Langmuir, (b) Freundlich, (c) Temkin and (d) $\mathrm{D}-\mathrm{R}$ equations (TBBPA $=2,5,10,20$ and $30 \mathrm{mg} \mathrm{L}^{-1}, \mathrm{MGAC}=150 \mathrm{mg} \mathrm{L}^{-1}, T=20,30$ and $40{ }^{\circ} \mathrm{C}, \mathrm{pH}=9.0, t=10 \mathrm{~h}$ ). 
Table 2 Isotherm parameters for TBBPA adsorption on MGAC

\begin{tabular}{|c|c|c|c|c|c|c|c|c|c|c|c|c|}
\hline $\begin{array}{l}T \\
\left({ }^{\circ} \mathrm{C}\right)\end{array}$ & \multicolumn{3}{|l|}{ Langmuir } & \multicolumn{3}{|c|}{ Freundlich } & \multicolumn{3}{|l|}{ Temkin } & \multicolumn{3}{|l|}{$D-R$} \\
\hline 30 & 110.2 & 0.3070 & 0.981 & 21.979 & 1.726 & 0.902 & 22.969 & 3.423 & 0.962 & 66.5 & 1.250 & 0.792 \\
\hline 40 & 75.5 & 0.6156 & 0.993 & 26.618 & 2.691 & 0.969 & 12.794 & 12.931 & 0.990 & 53.7 & 0.410 & 0.807 \\
\hline
\end{tabular}

Table 3 Thermodynamic parameters for TBBPA adsorption on the MGAC at different temperatures

\begin{tabular}{llll}
\hline $\begin{array}{l}T \\
\left({ }^{\circ} \mathrm{C}\right)\end{array}$ & $\begin{array}{l}\Delta G^{0}(\mathrm{~kJ} \\
\left.\mathrm{mol}^{-1}\right)\end{array}$ & $\begin{array}{l}\Delta H^{0}(\mathrm{~kJ} \\
\left.\mathrm{mol}^{-1}\right)\end{array}$ & $\begin{array}{l}\Delta S^{0}(\mathrm{~J} \\
\left.\mathrm{mol}^{-1} \mathrm{~K}^{-1}\right)\end{array}$ \\
\hline 20 & -18.79 & -4.97 & 47.16 \\
30 & -19.26 & & \\
40 & -19.76 & & \\
\hline
\end{tabular}

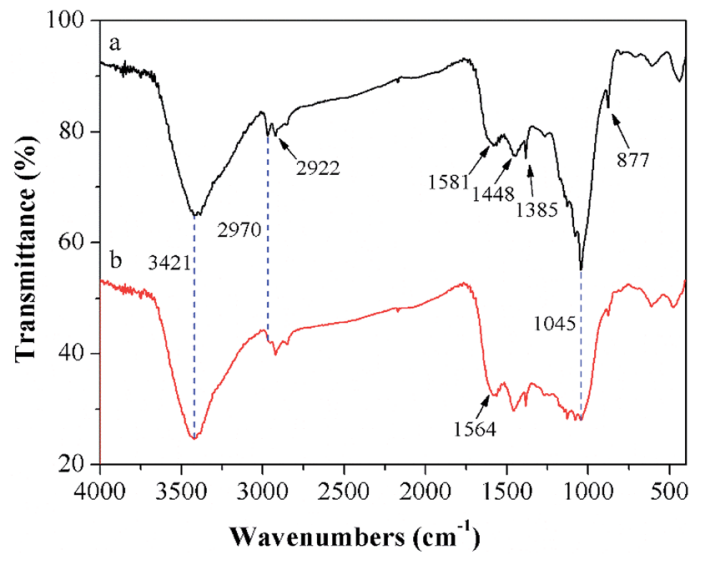

Fig. 8 FTIR spectra of the MGAC (a) and the MGAC with TBBPA adsorbed (b).

removal process. According to the fitting results, the saturated adsorption capacity of the MGAC for TBBPA came up to $110.7 \mathrm{mg} \mathrm{g}^{-1}$, which was significantly superior to common commercial activated carbons (Fig. S3 $\dagger$ ). Moreover, the $n$ values of Freundlich model ranged from 1.726 to 2.691 indicated that this adsorption process was feasible and all adsorption isotherms were nonlinear, ${ }^{25}$ which could be attributed to the various functional groups on the MGAC and the different dominant interactions like hydrogen bonding in the adsorption process.

In addition, the thermodynamics parameters including $\Delta G^{0}$, $\Delta H^{0}$ and $\Delta S^{0}$ were also calculated and listed in Table 3. The results showed that $\Delta G^{0}$ was negative at different temperatures, indicating that the TBBPA adsorption using the MGAC was a thermodynamically feasible and spontaneous process. The negative $\Delta H^{0}$ illustrated that the adsorption was an exothermic process, which would present preferable performance with lower temperature.

\subsection{Adsorption mechanism of the MGAC for TBBPA}

According to previous studies, diversified adsorption impetuses to remove TBBPA using activated carbons had been proposed, including electrostatic interactions, hydrogen bonding, $\pi-\pi$ interactions, hydrophobic effect. ${ }^{32}$ To further understand the adsorption mechanisms of TBBPA onto the MGAC surface, the FTIR spectra of the MGAC before and after adsorption were compared (Fig. 8). In addition, the peaks corresponding functional groups were demonstrated which certified the existence of multifarious oxygen-containing groups on the MGAC surface (Table 4). It is worth to highlight the change of the band at $1045 \mathrm{~cm}^{-1}$ after adsorption, which could be ascribed to the combination between TBBPA and -OH groups on the MGAC surface via hydrogen bonding. ${ }^{17}$ The disappearance of band at $2922 \mathrm{~cm}^{-1}$ and shift of band at $1581 \mathrm{~cm}^{-1}$ to $1564 \mathrm{~cm}^{-1}$ after adsorption could be attributed to the $\pi-\pi$ interactions between TBBPA and the MGAC resulting in the variations of electron density and on the MGAC surface. ${ }^{18}$

Based on these results, the possible adsorption processes of TBBPA onto the MGAC surface were further proposed (Fig. 9). It was deduced that the $\pi-\pi$ interactions probably played a significant role in the adsorption due to the benzene rings and

Table 4 The peaks of FTIR spectra corresponding to functional groups

\begin{tabular}{lll}
\hline Peak $\left(\mathrm{cm}^{-1}\right)$ & Characteristic vibration & Functional groups \\
\hline 3421 & O-H vibration & $-\mathrm{OH}^{41}$ \\
2970 & O-H vibration & $-\mathrm{OH}^{42}$ \\
2922 & C-H stretching vibration & $-\mathrm{CH}_{x}{ }^{17}$ \\
1581 & Stretching vibrations of aromatic rings & Aromatic rings $^{43}$ \\
1448 & Stretching vibrations of aromatic rings & Aromatic rings $^{43}$ \\
1385 & O-H bending vibration & $-\mathrm{OH}^{17}$ \\
1045 & C-O stretching vibration & ${\mathrm{C}=\mathrm{O}^{44}}^{43}$ \\
877 & $\mathrm{C}=\mathrm{C}$ vibration & $\mathrm{C}=\mathrm{C}^{17}$
\end{tabular}




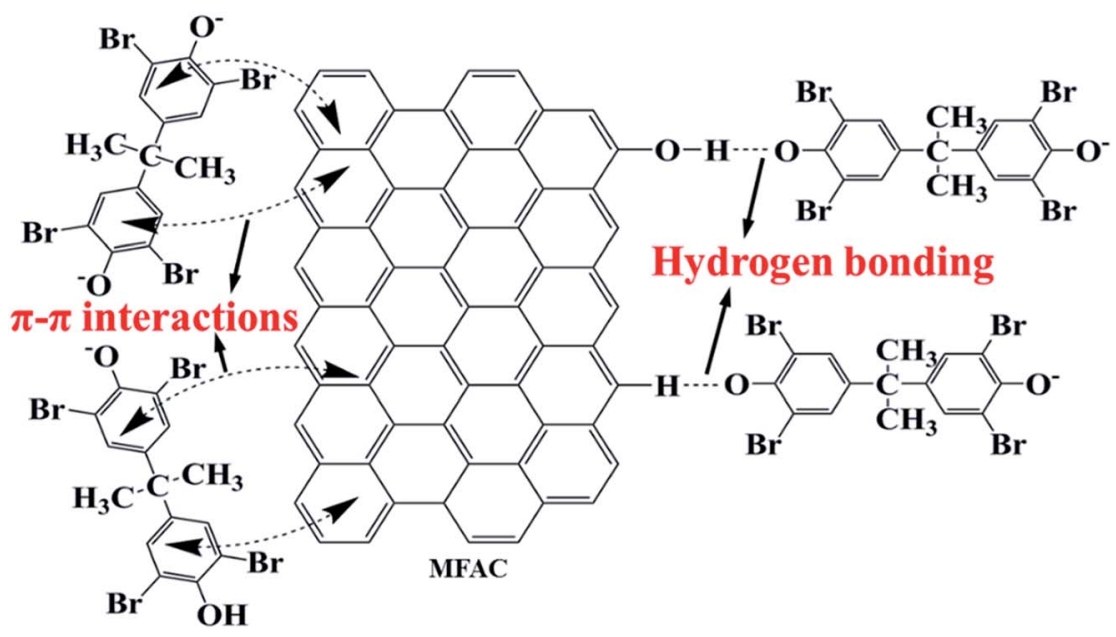

Fig. 9 Schematic diagram for the adsorption of TBBPA using the MGAC.

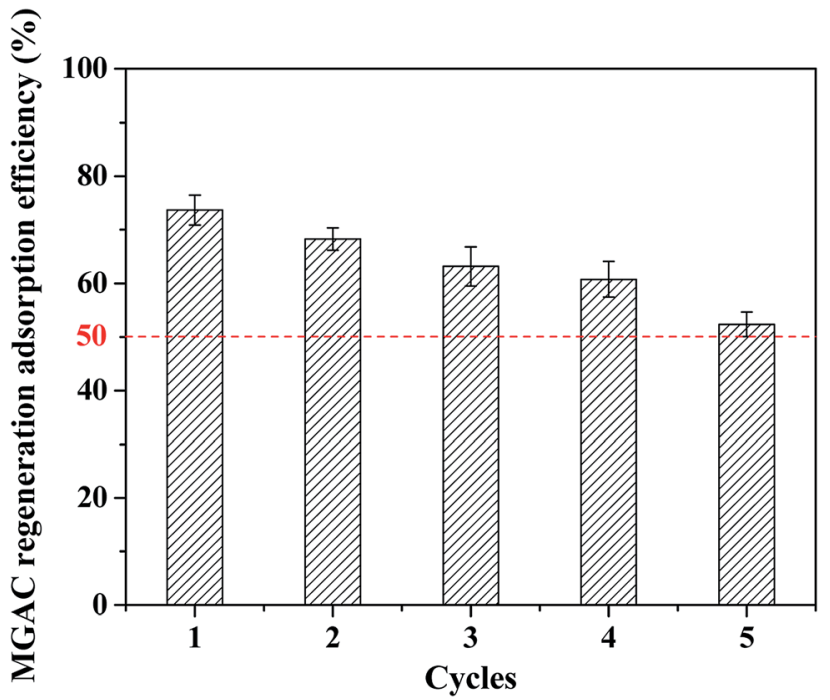

Fig. 10 Regeneration of $M G A C$ by $5 \% \mathrm{NaOH}$ (TBBPA $=10 \mathrm{mg} \mathrm{L}^{-1}$, $M G A C=150 \mathrm{mg} \mathrm{L}^{-1}, T=30^{\circ} \mathrm{C}, \mathrm{pH}=9.0, t=10 \mathrm{~h}$ ).

-OH groups on the MGAC surface as the electron-donating functional groups might enhance the $\pi$-donating strength of the aromatic rings of TBBPA. In addition, hydrogen bonding also generated significant influence on the adsorption for the MGAC could act as hydrogen bond donors dependent on the $-\mathrm{OH}$ groups and aromatic rings on the surface, resulting in a better adsorption capacity. Besides, there may be electron exchange or covalent bond between adsorbents and adsorbates according to the results of kinetic model analysis.

\subsection{Regeneration of MGAC}

In order to extend the lifetime of the MGAC, the used MGAC was regenerated using $\mathrm{NaOH}$ solution as the eluent and then subjected to the TBBPA adsorption again. As shown in Fig. 10, the spent MGAC was successfully regenerated by alkaline and retained over $50 \%$ of its initial capacity for TBBPA after 5 successive adsorption-regeneration cycles, which could be attributed to a decrease in the number of adsorption sites. The results nevertheless proved that the MGAC based the long-root Eichhornia crassipes could be used repeatedly in an adsorptiondesorption cycle, which was an essential advantage with regard to the practical applications. In addition, many regenerative methods have been developed recently, such as pyrolysis, which is versatile because it can decompose various organic contaminants. Studies have confirmed that TBBPA can be pyrolyzed at 280-900 ${ }^{\circ} \mathrm{C},{ }^{45}$ which provides a new efficient strategy for the MGAC regeneration in the future application.

\section{Conclusion}

In this study, the MGAC prepared from the waste long-root Eichhornia crassipes plants, had abundant functional groups on its surface, which could effectively remove TBBPA, indicating that it had good adsorption performance for organic pollutants. Moreover, it was confirmed that $\pi-\pi$ interaction and hydrogen bonding were the major impetuses for the adsorption on the basis of multiple characterization and experimental analysis. The in-depth understanding of the adsorption properties and mechanism will offer valuable counsel for the application of the MGAC in the wastewater treatment and provide a new way for the resource utilization of long-root Eichhornia crassipe in the future.

\section{Conflicts of interest}

There are no conflicts to declare.

\section{Acknowledgements}

This work was sponsored by the National Natural Science Foundation of China (41771513, 51704121, 41001316, 51108262), Major Science and Technology Program for Water Pollution Control and Treatment in China (2017ZX07202006) 
and Shanghai Pujiang Program (16PJ1404800), National Key R\&D Program of China (2018YFC1901000).

\section{References}

1 J. L. Lyche, C. Rosseland, G. Berge and A. Polder, Environ. Int., 2015, 74, 170-180.

2 J. Xu, Y. Zhang, C. Guo, Y. He, L. Li and W. Meng, Environ. Toxicol. Chem., 2013, 32, 2249-2255.

3 J. Xiong, T. An, C. Zhang and G. Li, Environ. Geochem. Health, 2015, 37, 457-473.

4 Y. Yang, L. Lu, J. Zhang, Y. Yang, Y. Wu and B. Shao, J. Chromatogr. A, 2014, 1328, 26-34.

5 A. H. Feng, S. J. Chen, M. Y. Chen, M. J. He, X. J. Luo and B. X. Mai, Mar. Pollut. Bull., 2012, 64, 919-925.

6 Z. Lu, R. J. Letcher, S. Chu, J. J. H. Ciborowski, G. Douglas Haffner, K. G. Drouillard, S. L. MacLeod and C. H. Marvin, J. Great Lakes Res., 2015, 41, 808-817.

7 F. T. Li and T. Yang, Automatica, 2017, 78, 223-230.

8 Y. Grosse, D. Loomis, K. Z. Guyton, F. El Ghissassi, V. Bouvard, L. Benbrahim-Tallaa, H. Mattock and K. Straif, Lancet Oncol., 2016, 17, 419-420.

9 Y. Ji, D. Kong, J. Lu, H. Jin, F. Kang, X. Yin and Q. Zhou, J. Hazard. Mater., 2016, 313, 229-237.

10 Y. Bao and J. Niu, Chemosphere, 2015, 134, 550-556.

11 Q. Huang, W. Liu, P. Peng and W. Huang, Chemosphere, 2013, 92, 1321-1327.

12 Q. Huang, W. Liu, P. Peng and W. Huang, J. Hazard. Mater., 2013, 262, 634-641.

13 M. Altarawneh and B. Z. Dlugogorski, J. Phys. Chem. A, 2014, 118, 9338-9346.

14 L. Zhou, L. Ji, P. C. Ma, Y. Shao, H. Zhang, W. Gao and Y. Li, J. Hazard. Mater., 2014, 265, 104-114.

15 F. Tong, X. Gu, C. Gu, R. Ji, Y. Tan and J. Xie, Sci. Total Environ., 2015, 536, 582-588.

16 I. I. Fasfous, E. S. Radwan and J. N. Dawoud, Appl. Surf. Sci., 2010, 256, 7246-7252.

17 J. Shen, G. Huang, C. An, X. Xin, C. Huang and S. Rosendahl, Bioresour. Technol., 2018, 247, 812-820.

18 Y. Zhang, Y. Tang, S. Li and S. Yu, Chem. Eng. J., 2013, 222, 94-100.

19 Z. Sun, Y. Yu, L. Mao, Z. Feng and H. Yu, J. Hazard. Mater., 2008, 160, 456-461.

20 Y. Zhang, L. Jing, X. He, Y. Li and X. Ma, J. Ind. Eng. Chem., 2015, 21, 610-619.

21 F. Cao, C. Lian, J. Yu, H. Yang and S. Lin, Bioresour. Technol., 2019, 276, 211-218.

22 S. Lin, H. Yang, Z. Na and K. Lin, Chemosphere, 2017, 192, 258.

23 S. Lin, G. Wang, Z. Na, D. Lu and Z. Liu, Chem. Eng. J., 2012, 183, 365-371.
24 V. Srihari and A. Das, Desalination, 2008, 225, 220-234.

25 W. Wang, S. Deng, D. Li, L. Ren, D. Shan, B. Wang, J. Huang, Y. Wang and G. Yu, Chem. Eng. J., 2018, 332, 286-292.

26 M. Song, B. Jin, R. Xiao, L. Yang, Y. Wu, Z. Zhong and Y. Huang, Biomass Bioenergy, 2013, 48, 250-256.

27 A. Ould-Idriss, M. Stitou, E. M. Cuerda-Correa, C. FernándezGonzález, A. Macías-García, M. F. Alexandre-Franco and V. Gómez-Serrano, Fuel Process. Technol., 2011, 92, 261-265.

28 J. Wang, T.-L. Liu, Q.-X. Huang, Z.-Y. Ma, Y. Chi and J.-H. Yan, Fuel Process. Technol., 2017, 162, 13-19.

29 Z. Gong, S. Li, J. Ma and X. Zhang, Sep. Purif. Technol., 2016, 157, 131-140.

30 T. Malkoske, Y. Tang, W. Xu, S. Yu and H. Wang, Sci. Total Environ., 2016, 569-570, 1608-1617.

31 A. Kalra, N. Tugcu, S. M. Cramer and S. Garde, J. Phys. Chem. B, 2001, 105, 6380-6386.

32 M. Kah, G. Sigmund, F. Xiao and T. Hofmann, Water Res., 2017, 124, 673-692.

33 Y. Y. Lv, X. H. Yu, S. T. Tu, J. Y. Yan and E. Dahlquist, Appl. Energy, 2012, 97, 283-288.

34 L. H. Jiang, Y. G. Liu, G. M. Zeng, F. Y. Xiao, X. J. Hu, X. Hu, H. Wang, T. T. Li, L. Zhou and X. F. Tan, Chem. Eng. J., 2016, 284, 93-102.

35 Y. Zhang, Y. Tang, S. Li and S. Yu, Chem. Eng. J., 2013, 222, 94-100.

36 L. Shuai, P. Wu, M. Chen, L. Yu, C. Kang, N. Zhu and D. Zhi, Environ. Pollut., 2017, 228, 277-286.

37 S. K. Behera, S. Y. Oh and H. S. Park, J. Hazard. Mater., 2010, 179, 684-691.

38 K. Qiang, H. Xiao, S. Li and M. M. Sheng, Process Saf. Environ. Prot., 2017, 112, 254-264.

39 L. Liu, S. Hu, G. Shen, U. Farooq, W. Zhang, S. Lin and K. Lin, Chemosphere, 2018, 196, 409-417.

40 T. Depci, A. R. Kul and Y. Önal, Chem. Eng. J., 2012, 200-202, 224-236.

41 N. M. A. Al-Lagtah, A. a. H. Al-Muhtaseb, M. N. M. Ahmad and Y. Salameh, Microporous Mesoporous Mater., 2016, 225, 504-514.

42 M. A. Islam, M. J. Ahmed, W. A. Khanday, M. Asif and B. H. Hameed, Ecotoxicol. Environ. Saf., 2017, 138, 279-285.

43 H. Deng, G. Li, H. Yang, J. Tang and J. Tang, Chem. Eng. J., 2010, 163, 373-381.

44 K.-L. Chang, J.-F. Hsieh, B.-M. Ou, M.-H. Chang, W.-Y. Hseih, J.-H. Lin, P.-J. Huang, K.-F. Wong and S.-T. Chen, Sep. Sci. Technol., 2012, 47, 1514-1521.

45 M. Altarawneh, A. Saeed, M. Al-Harahsheh and B. Z. Dlugogorski, Prog. Energy Combust. Sci., 2019, 70, 212-259. 